\title{
ANÁLISE DOS FUNDAMENTOS DO MODELO VALUE-BASED HEALTH CARE DELIVERY À LUZ DAS TEORIAS DE ESTRATÉGIA
}

\author{
ANALYSIS OF THE VALUE-BASED HEALTH CARE DELIVERY MODEL IN LIGHT OF STRATEGY \\ THEORIES
}

ANÁLISIS DE LOS FUNDAMENTOS DEL MODELO VALUE-BASED HEALTH CARE DELIVERY A LA LUZ DE LAS TEORÍAS DE LA ESTRATEGIA

\author{
Germany Gonçalves Veloso \\ Doutor \\ Fundação Getúlio Vargas \\ germanyveloso@gvmail.br
}

Rodrigo Bandeira-de-Mello

Doutor

Fundação Getúlio Vargas rodrigo.bandeira.demello@fgv.br

Ana Maria Malik

Doutora

Fundação Getúlio Vargas ana.malik@fgv.br

Submetido em: $12 / 01 / 2012$

Aprovado em: 19/02/2014

\section{RESUMO}

O setor de saúde, globalmente, apresenta problemas relacionados aos seus custos, qualidade e acesso. Porter e Teisberg $(2004,2006)$ propuseram modelo de gestão específico para a administração estratégica na área, o Value-Based Health Care Delivery (VBHCD). O modelo teve relativa repercussão e vem influenciando muitos atores no setor. Contudo, o modelo vem sendo aceito sem o devido questionamento de seus fundamentos e consistência com a teoria em estratégia. O presente trabalho busca, por meio de um ensaio teórico, analisar o modelo proposto à luz de paradigmas gerais da estratégia empresarial como, por exemplo, o modelo de Porter e a Visão Baseada em Recursos (RBV). Inicialmente, o artigo sintetiza as explicações teóricas do modelo porteriano clássico e da RBV. Em seguida, o VBHCD é examinado comparado a essas explicações, buscando-se relações e, eventualmente, contradições. Conclui-se que o modelo VBHCD não se alinha integralmente a nenhum corpo teórico isoladamente. Mesmo sendo proposto por Porter, parte de seus fundamentos advém, na verdade, de outras correntes teóricas. Esta abordagem integradora de teorias concorrentes, apesar de presente na literatura, ainda apresenta dificuldades e barreiras, um aspecto que não está explícito no modelo.

PALAVRAS-ChAVE: Estratégia Competitiva. Saúde. Valor. Desempenho.

\section{ABSTRACT}

Globally, the health sector presents problems related to cost, quality and access. Porter and Teisberg $(2004,2006)$ proposed a specific management model for strategic administration in this sector - Value-Based 
Health Care Delivery (VBHCD). This model has been adopted by many actors in the health care sector. However, it has been accepted without a proper analysis of its bases and consistency with strategic theory. This theoretical essay analyzes the proposed model in light of the general paradigms of business strategy, such as Porter's model and the Resource Based View. Initially, the article summarizes the theoretical explanations of Port's classic model and RBV. Next, the VBHCD is examined, comparing it with these explanations, and looking for any links and contradictions. We conclude that the VBHCD model is not fully aligned with any one specific theory. Although proposed by Porter, part of its rationale actually stems from other theoretical perspectives. This integrative approach of concurrent theories, although it is presented in the literature, still faces difficulties and barriers, an aspect that is not clear in the model.

KEYWORDS: Competitive Strategy. Health Care. Value. Performance.

\section{RESUMEN}

Globalmente, el sector de salud presenta problemas relacionados a sus costos, calidad y acceso. Porter y Teisberg $(2004,2006)$ propusieron un modelo de gestión específico para la administración estratégica en el área, el Value-Based Health Care Delivery (VBHCD). Este modelo tuvo relativa repercusión y está ejerciendo influencia sobre muchos actores en el sector. Sin embargo, el modelo viene siendo aceptado sin el debido cuestionamiento de sus fundamentos y consistencia con la teoría en estrategia. El presente trabajo se propone, por medio de un ensayo teórico, analizar el modelo propuesto a la luz de paradigmas generales de la estrategia empresarial, como por ejemplo el modelo de Porter y la Visión Basada en los Recursos (RBV). Inicialmente, el artículo sintetiza las explicaciones teóricas del modelo porteriano clásico y de la RBV. A continuación, el VBHCD es examinado en comparación a esas explicaciones, buscándose relaciones y eventualmente contradicciones. Se concluye que el modelo VBHCD no se alinea integralmente a ningún cuerpo teórico de forma aislada. Aun siendo propuesto por Porter, parte de sus fundamentos provienen, en verdad, de otras corrientes teóricas. Este abordaje integrador de teorías concurrentes, a pesar de estar presente en la literatura, todavía presenta dificultades y barreras, un aspecto que no está explícito en el modelo.

PALABRAS CLAVE: Estrategia Competitiva. Salud. Valor. Desempeño.

\section{INTRODUÇÃO}

Há muito se debate sobre a dificuldade de aplicação dos modelos de Administração na área de saúde. Alguns autores afirmam que a área em si não tem sido alvo de investigação (PAIM; TEIXEIRA, 2006). Já para outros, ela apresenta uma dificuldade para definição de seus limites (ALMEIDA, C., 2004), possui limites bastante difusos (NOVAES, 2004), é caracterizada por diversidade e complexidade (ALMEIDA, M., 2004) ou "não tem uma estrutura conceitual bem definida, é marcadamente multidisciplinar, desenvolvida por múltiplos atores (academia, hospitais, governo)" (POLANCZIK, 2010, p. 3). Além disso, há o questionamento quanto a se existem especificidades da área, ou se seria desejável aplicar os conhecimentos já consagrados nos demais setores (MISOCZKY et al., 2009).

Porém pesquisadores habitualmente ligados à pesquisa em Administração começam a propor modelos aplicados à saúde. Em meados da primeira década do século XXI, foi proposto o modelo de Value-Based Health Care Delivery (VBHCD) por Porter e Teisberg $(2004,2006)$. O modelo tem gerado aplicações no setor, reações de diversos autores como, por exemplo, Mintzberg (2012), Christensen, Grossman e Hwang (2009) e Kotler, Shalowitz e Stevens (2008), além de centenas de outras citações para seus principais artigos.

Apesar dessa influência em termos de publicações e também de intervenções na prática do setor, não há na literatura um exame dos fundamentos teóricos do modelo. O presente artigo supre essa lacuna e oferece uma análise do modelo, expondo relações e contradições teóricas à luz das principais correntes teóricas em estratégia e vantagem competitiva. 


\section{REVISÃO DA LITERATURA}

Inicialmente apresentam-se sínteses das explicações da vantagem competitiva pelo paradigma porteriano e pela Visão Baseada em Recursos (VBR), tendo em vista que são apontadas como as linhas mais influentes no debate de Estratégia. Em seguida, uma síntese sobre aspectos relevantes do setor de saúde, do ponto de vista da prática e do estudo de estratégia empresarial. Posteriormente, examina-se o Modelo do VBHCD à luz dessas explicações e por meio de dimensões de análise definidas a partir da revisão dessa literatura. Cabe lembrar que o termo modelo é utilizado aqui no sentido de teoria, como fazem algumas autoridades no desenvolvimento de teorias (DUBIN, 1978).

\section{O paradigma porteriano}

O paradigma porteriano, conhecido também como "Visão Baseada no Mercado" (VBM), considera que a vantagem competitiva é uma consequência do posicionamento da empresa, que é derivada da estrutura da indústria na qual a firma opera. O posicionamento refere-se a como a empresa irá entregar valor superior. Ele guiará a forma como as atividades serão organizadas internamente na organização (PORTER, 1979, 1981, 1986). São pressupostos implícitos da VBM, uma estrutura de mercado dada e as atividades da empresa consideradas como passíveis de livre configuração, ou seja, este é o ambiente mais apropriado para sua aplicação.

Esta visão iniciou-se com Caves e Porter (1977), que desenvolveram uma perspectiva teórica a partir da Organização Industrial. Eles consideram que a variação de performance verificada dentro das indústrias é devida não somente ao poder de mercado das firmas e à sorte, mas também às decisões estratégicas que essas empresas fazem. Porter $(1979,1981)$ apresentou sua teoria para explicar a determinação da rentabilidade de uma empresa e como ela variava dependendo da estrutura de indústria à qual pertence. Ou seja, para ele, a estrutura da indústria determina o comportamento das empresas (sua estratégia) que, por sua vez, determina o desempenho das empresas no mercado. Essa teoria foi construída baseada nos conceitos de grupos estratégicos e de barreiras de mobilidade e provê explicações para diferenças persistentes entre as estratégias competitivas das empresas de uma mesma indústria.

Porter destaca sua descrição dos determinantes fundamentais da rentabilidade de uma empresa: (1) as características comuns da indústria; (2) as características do grupo estratégico e (3) a posição da empresa dentro de seu grupo estratégico (PORTER, 1986). O quadro de referência para análise da estrutura da indústria é o denominado modelo das cinco forças competitivas: (1) ameaça dos novos entrantes; (2) poder de barganha dos compradores; (3) poder de barganha dos fornecedores; (4) ameaça dos substitutos; e (5) rivalidade entre os competidores (PORTER, 1986, 2008b). Outro aspecto importante da Teoria de Porter é a importância da complementaridade entre as diversas atividades organizadoras da empresa, a fim de aumentar ainda mais o trade-off da escolha de determinado posicionamento estratégico, de aumentar as barreiras de mobilidade e de garantir a sustentabilidade da performance (PORTER, 1996). No mesmo artigo (PORTER, 1996), que por sinal, praticamente é uma resposta às muitas críticas colocadas em relação ao seu modelo de estratégia, Porter reitera que o importante em estratégia é a combinação das atividades da empresa no sentido de obter o posicionamento escolhido (em oposição à busca da efetividade que, segundo ele, é a excelência em atividades individuais).

Porter (1986) também desenvolveu o conceito de Cadeia de Valor, cujo objetivo é detalhar as atividades da empresa que têm relevância estratégica, a fim de possibilitar o entendimento do comportamento dos seus custos e dos seus potenciais de diferenciação. Cadeia de Valor é definida como uma representação de todas as atividades realizadas por uma determinada empresa para projetar, produzir, comercializar, entregar e sustentar seu produto. De acordo com esse modelo, as atividades da empresa podem ser divididas em dois tipos: atividades primárias, aquelas envolvidas diretamente no fluxo de produtos para o consumidor, como logística, operações, marketing e vendas e serviços pós-vendas; e atividades de apoio (ou secundárias), não envolvidas diretamente nesse fluxo, como compra de materiais, gerenciamento de tecnologia, de recursos humanos e de infraestrutura da empresa.

Alguns trabalhos empíricos realizados no Brasil são úteis para demonstrar a aplicação do Modelo de Posicionamento de Porter na realidade local: Bandeira-de-Mello e Marcon (2004) evidenciaram 
diferenças significativas de variação das rentabilidades das firmas (uma medida para eficácia das estratégias de posicionamento) em torno da média ROE, em relação a dois grupos de diferentes setores. Pinho e Silva (2001) demonstraram a adequação do modelo de grupos estratégicos para descrever e explicar a realidade da indústria farmacêutica no Brasil. Para Sehnem, Lazzarotti e Bandeira-De-Mello (2010), o modelo de Porter teve seu auge no Brasil no final da década de 90, mas sua utilização apresenta um declínio, provavelmente em virtude da popularidade da corrente teórica da RBV. Os autores evidenciam também que a utilização do paradigma porteriano em pesquisas no Brasil tem sido feita de forma acrítica para descrever a realidade da estratégia no país.

\section{Visão da firma baseada em recursos}

A VBR tem se tornado uma perspectiva influente no debate sobre Estratégia Empresarial, principalmente após alguns autores associarem o fato de o desempenho das empresas se correlacionarem mais com suas características internas do que com a indústria em que atuam (BRITO; VASCONCELOS, 2009; HAWAWINI; SUBRAMANIAN; VERDIN, 2003; MCGAHAN; PORTER, 1997; MISANGYI et al., 2006; RUMELT, 1991). No Brasil, diversos estudos têm analisado, entre outros aspectos, o efeito da heterogeneidade das empresas (BANDEIRA-DE-MELLO; MARCON, 2005) e as taxas de crescimento das organizações (BRITO, VASCONCELOS, 2009), por exemplo. Apesar de críticas com relação à característica ateórica de estudos de decomposição de variância, o fato de a indústria ser menos importante do que a firma para discriminar desempenhos coloca a atenção nos aspectos internos como fonte de vantagem competitiva.

Há um número crescente de estudos mostrando a utilização da abordagem da VBR para responder aos desafios pela busca da vantagem competitiva sustentável (RAMOS-RODRIGUES; RUIZ-NAVARRO, 2004; SCHROEDER; BATES; JUNTTILA, 2002). A VBR defende que o conjunto de recursos e competências único de uma empresa é a sua maior fonte de vantagem competitiva. Para ela, as diferenças de desempenho entre as empresas ocorrem fundamentalmente em função da heterogeneidade e da imperfeita mobilidade (entre empresas) dos recursos e das capacidades que elas detêm (BARNEY, 1991) - e que são pressupostos da VBR -, quer eles sejam adquiridos ou desenvolvidos internamente (DIERICKX; COOL, 1989). Esses recursos e capacidades devem ter as seguintes características para serem considerados como tal (BARNEY, 1991, 1999):

- Valor: recursos são valiosos quando permitem à empresa conceber ou implementar estratégias que melhoram sua eficiência ou efetividade. Dito de outra forma, serem capazes de aproveitar oportunidades ou neutralizar ameaças no ambiente da empresa;

- Raridade: devem ser raros entre os atuais e os potencias concorrentes da empresa. O número de empresas que devem possuir um particular recurso deve ser inferior àquele que geraria uma dinâmica de competição perfeita, segundo Peteraf e Bergen (2003);

- Imitabilidade Imperfeita. A dificuldade de imitação advém de: (1) condições históricas únicas, isto é, do caminho no tempo e no espaço que a empresa atravessou durante aquisição ou desenvolvimento de seus recursos; (2) causa ambígua, isto é, quando a ligação entre os recursos da empresa e sua vantagem competitiva é pouco compreendida; e (3) recurso social complexo, o que ocorre quando o fenômeno está além da capacidade de gerenciamento sistemático da empresa, o que inclui reputação da empresa, seus relacionamentos e sua cultura organizacional; e finalmente,

- Substituibilidade Imperfeita: não deve existir recurso estrategicamente equivalente que seja também valioso, mas não raro ou imperfeitamente imitável.

Peteraf (1993) lembra ainda que, além da heterogeneidade e da imperfeita imobilidade, garantir uma baixa competição ex-ante, enquanto se adquire ou estabelece sua posição de recursos, bem como uma igualmente baixa competição ex-post, também são fatores importantes para a vantagem competitiva. A baixa competição ex-ante possibilita que as rendas provenientes dos recursos não sejam superadas pelos custos de sua aquisição ou desenvolvimento. A baixa competição ex-post assegura que as rendas não se dissipem.

Enquanto no paradigma porteriano o lócus da vantagem competitiva é externo (explorar os trade-offs da indústria), para a RBV o lócus é interno (o valor de seus recursos). Pesquisadores da 
VBR fazem uma análise interna das forças e das fraquezas como ponto de partida para a formulação estratégica, e autores da VBM partem das ameaças e das oportunidades.

Existem várias críticas em relação à RBV. Um intenso debate é travado em relação a aspectos possivelmente tautológicos dela (BARNEY, 2001; PRIEM; BUTLER, 2001a, 2001b). Para outros, a VBR é uma corrente estática. Em relação a estes últimos, pesquisadores desenvolveram os conceitos de capacidades e de capacidades dinâmicas, como recursos da firma baseados no conhecimento. Essa divisão da VBR é considerada muitas vezes um modelo à parte, aplicável a ambientes de alta complexidade e mudanças constantes (VASCONCELOS; CYRINO, 2000). Outros ainda o consideram como um ramo da VBR, a Knowledge-based View (KBV) (ACEDO; BARROSO; GALAN, 2006). De qualquer forma, esta visão está mais dedicada ao estudo da criação ou do desenvolvimento de recursos geradores de vantagem competitiva, principalmente daqueles baseados no conhecimento em ambientes mais dinâmicos (DOSI; NELSON; WINTER, 2000).

A fonte de vantagem competitiva na visão da KBV é o desenvolvimento de competências baseadas em conhecimento por meio de um processo de contínuo aprendizado organizacional. A vantagem competitiva das empresas, nesta perspectiva, se origina em combinações difíceis de imitar de capacidades gerenciais, organizacionais e tecnológicas. Essas capacidades estão imbricadas nos processos (formas de coordenar e combinar) e na estrutura organizacional das empresas. A vantagem competitiva das empresas também é formatada pelos seus demais recursos específicos e pelas suas alternativas de estratégias disponíveis (TEECE; PISANO; SHUEN, 1997).

Hamel e Prahalad (1994) definiram competências como um conjunto de habilidades e tecnologias de uma empresa; competência central representaria uma somatória de aprendizados ocorridos cruzando fronteiras de equipes e unidades operacionais ou funcionais, e, portanto, raramente encontrada confinada a uma unidade ou equipe. Em 1990, Prahalad e Hamel definiram competências centrais como o aprendizado coletivo da organização, especialmente como coordenar diversas habilidades produtivas e integrar múltiplos fluxos tecnológicos (PRAHALAD; HAMEL, 1990). Segundo os autores, três características são necessárias para que uma competência seja considerada central (HAMEL; PRAHALAD, 1994): (a) valor para o cliente: uma competência central proporciona ao cliente um valor percebido por ele como central; (b) diferenciação em relação à concorrência; e (c) "extendabilidade": uma competência central tem papel na abertura de novos mercados e oportunidades.

Segue o Quadro 1 com a síntese sobre as principais características dos modelos descritos anteriormente:

Quadro 1 - Comparação entre os principais modelos de vantagem competitiva

\begin{tabular}{|c|c|c|c|}
\hline & Paradigma Porteriano & RBV & KBV \\
\hline $\begin{array}{l}\text { Principais } \\
\text { Autores }\end{array}$ & Bain, Mason, Porter & $\begin{array}{l}\text { Penrose, Peteraf, } \\
\text { Wernerfelt, Barney }\end{array}$ & $\begin{array}{l}\text { Nelson e Winter, Teece, } \\
\text { Kogut }\end{array}$ \\
\hline Firma & $\begin{array}{l}\text { Conjunto de atividades } \\
\text { complementares ou sistema } \\
\text { de atividades (compras, } \\
\text { logística, vendas, pós-vendas, } \\
\text { etc.). }\end{array}$ & Feixe de recursos & $\begin{array}{l}\text { Conjunto de recursos baseado } \\
\text { no conhecimento (capacidades, } \\
\text { rotinas, competências). }\end{array}$ \\
\hline Mercado & $\begin{array}{l}\text { Estático. Mercado se ajusta } \\
\text { até chegar ao equilíbrio. } \\
\text { Lucro tende a zero. }\end{array}$ & $\begin{array}{l}\text { Estático. Importância na } \\
\text { proteção dos recursos. } \\
\text { Fonte de mudança } \\
\text { exógena. }\end{array}$ & $\begin{array}{l}\text { Dinâmico. Criação por meio } \\
\text { da inovação. Não tende ao } \\
\text { equilíbrio. Fonte de mudança } \\
\text { endógena. Ênfase na criação, } \\
\text { na renovação e não na proteção } \\
\text { de recursos. }\end{array}$ \\
\hline Estratégia & $\begin{array}{l}\text { Estratégia envolve escolha } \\
\text { em face de trade-offs para } \\
\text { posicionarafirma no mercado. } \\
\text { A Estratégia (escolhas) tem } \\
\text { papel importante para o } \\
\text { desempenho. }\end{array}$ & $\begin{array}{l}\text { Definida a partir dos } \\
\text { recursos da firma. } \\
\text { Estratégia secundária } \\
\text { em relação à base de } \\
\text { recursos, que impõem } \\
\text { restrições à escolha das } \\
\text { estratégias. }\end{array}$ & $\begin{array}{l}\text { Idem à RBV, contudo com } \\
\text { ênfase nas estratégias de } \\
\text { inovação de desenvolvimento } \\
\text { de novos produtos. }\end{array}$ \\
\hline
\end{tabular}




\begin{tabular}{|c|c|c|c|}
\hline $\begin{array}{l}\text { Agência dos } \\
\text { Executivos }\end{array}$ & $\begin{array}{l}\text { Agência é alta. Cabe ao gestor } \\
\text { escolher em quais dimensões } \\
\text { competitivas a empresa irá } \\
\text { atuar. Escolha limitada pela } \\
\text { estrutura da indústria. }\end{array}$ & $\begin{array}{l}\text { Nível de agência baixo. } \\
\text { o gestor não consegue } \\
\text { identificar ex-ante as } \\
\text { causas do sucesso } \\
\text { da empresa, devido à } \\
\text { ambiguidade causal e à } \\
\text { complexidade social. A } \\
\text { dependência de recursos } \\
\text { importantes à história da } \\
\text { firma também confere } \\
\text { menos agência ao gestor. }\end{array}$ & $\begin{array}{l}\text { Busca por inovações tem } \\
\text { característica de "tentativa } \\
\text { e erro", quando a empresa } \\
\text { equilibra processos de } \\
\text { "exploration/exploitation". } \\
\text { Nível de agência é baixo. }\end{array}$ \\
\hline $\begin{array}{l}\text { Fonte de } \\
\text { Vantagem } \\
\text { Competitiva }\end{array}$ & $\begin{array}{l}\text { D e f i n ição de u m } \\
\text { p os i c i o n a me nto e } \\
\text { consequente sistema de } \\
\text { atividades que entregue } \\
\text { valor superior. Consistência } \\
\text { (fit) entre as atividades e } \\
\text { os trade-offs é a fonte de } \\
\text { vantagem competitiva. }\end{array}$ & $\begin{array}{l}\text { Valor e raridade dos } \\
\text { recursos. }\end{array}$ & $\begin{array}{l}\text { Valor e raridade dos } \\
\text { recursos. }\end{array}$ \\
\hline $\begin{array}{l}\text { Sustentação } \\
\text { de Vantagem } \\
\text { competitiva }\end{array}$ & $\begin{array}{l}\text { Barreiras de mobilidade } \\
\text { d e c o r r e n t e s d o } \\
\text { comprometimento da empresa } \\
\text { com suas escolhas. Barreiras } \\
\text { cognitivas que desestimulam } \\
\text { os im itadores. Ga stos } \\
\text { irrecuperáveis impõem riscos } \\
\text { ao imitador, pois devem copiar } \\
\text { todo o sistema de atividades. A } \\
\text { consistência entre as atividades } \\
\text { e os trade-offs sustentam } \\
\text { vantagem competitiva. }\end{array}$ & \multicolumn{2}{|c|}{$\begin{array}{l}\text { Imitabilidade e substituibilidade imperfeitas. Mecanismos } \\
\text { complexidade social, ambiguidade causal e dependência } \\
\text { de condições históricas. }\end{array}$} \\
\hline $\begin{array}{l}\text { Unidade de } \\
\text { Análise }\end{array}$ & Grupo estratégico & Firma & Firma \\
\hline
\end{tabular}

\section{A ESTRATÉGIA EMPRESARIAL E A SAÚDE}

No Brasil, os gastos realizados com bens e serviços de saúde gira em torno de $8,4 \%$ do PIB (nos EUA, este valor gira em torno de $16 \%$, e nos países desenvolvidos, em geral acima de $10 \%$ ) e há previsão que estes números aumentem com o progressivo envelhecimento da população. Destes, $60,2 \%$ são feitos pelas famílias e 38,8\% pela administração pública (IBGE, 2012). Cerca de $70 \%$ dos hospitais brasileiros são privados (MINISTÉRIO DA SAÚDE, 2012). Entre 2000 e 2010, enquanto a população brasileira cresceu $12,3 \%$, o número de brasileiros com planos de saúde cresceu $48 \%$, passando de 30,7 para 45,6 milhões de usuários (IBGE, 2012).

Já em relação à prática e aos estudos acadêmicos na área de gestão em saúde, estes têm forte influência de paradigmas da área de Saúde Coletiva, e mais recentemente têm sofrido maior influência da ciência administrativa, em uma tendência que parece similar ao que ocorreu nos EUA a partir da década de 80 (SCOTT, 2003; SCOTT et al., 2000).

Do ponto de vista de estratégia empresarial, a característica mais relevante da saúde é que este é um setor altamente institucionalizado (ALEXANDER; D'AUNNO, 2003; SHORTELL; ZAJAC, 1990; LUKE; BEGUN; WALSTON, 2000; SCOTT, 2003; SCOTT et al., 2000). Um setor de saúde típico está inserido em um contexto de valores e normas sociais, além de um emaranhado de leis, normais éticas e profissionais, resoluções e agências regulatórias. Alguns exemplos destas instituições vão desde valores éticos e humanitários, como, por exemplo, não se conhece lugar no mundo em que seja aceitável negar atendimento de urgência em função da capacidade de pagamento do paciente (uma situação que envolve elevados riscos financeiros), até a restrição ao tipo de produtos (e 
preços) que planos de saúde podem oferecer, normas de propaganda e ética profissional, restrições à participação acionária em hospitais, reservas financeiras para planos de saúde, gastos obrigatórios de infraestrutura de hospitais, entre os inúmeros outros casos de obrigações e restrições. Não obstante tudo isso, pesquisas mostram que os cinquenta maiores hospitais e operadoras de planos de saúde do país têm rentabilidade comparável às maiores empresas do país (VELOSO; MALIK, 2010).

Em relação aos principais modelos teóricos de estratégia empresarial, vários autores mostram que estes apresentam poder explicativo e utilidade prescritiva no ambiente da saúde, porém são restringidos por forças institucionais (ALEXANDER; D'AUNNO, 2003; SHORTELL; ZAJAC, 1990; LUKE; BEGUN; WALSTON, 2000; LUKE; WALSTON, 2003; SCOTT, 2003; SCOTT et al., 2000). Elas efetivamente restringem as opções estratégicas àquelas consideradas legítimas. Além de determinar as condições, as forças institucionais também determinam sob quais critérios a competição ocorre na saúde, cujos aspectos ligados à qualidade - e secundariamente, à equidade - tomam maior importância (ALEXANDER; D'AUNNO, 2003; SHORTELL; ZAJAC, 1990; LUKE; BEGUN; WALSTON, 2000; LUKE; WALSTON, 2003; SCOTT, 2003; SCOTT et al., 2000).

\section{PROPOSIÇÕES E DIMENSÕES PARA ANÁLISE DO MODELO VBHCD}

A partir do referencial teórico descrito anteriormente, derivaram-se as seguintes proposições como base para análise do modelo em questão:

- Proposição 1 (P1): o VBHCD é um modelo estratégico para organizações de saúde que têm como medidas de desempenho, além de resultados econômico-financeiros, o valor para os pacientes.

- Proposição 2a (P2a): o VBHCD está alinhado ao modelo porteriano, ou seja, enfatiza, na concepção da estratégia empresarial, que a fonte e a sustentabilidade de desempenho superior advêm da escolha de um posicionamento no mercado.

- Proposição 2b (P2b): o VBHCD está alinhado à RBV, ou seja, enfatiza, na concepção da estratégia empresarial, que a fonte e a sustentabilidade de desempenho superior advêm de recursos internos da empresa que sejam valiosos, raros, de difícil imitação e substituibilidade.

- Proposição 2c (P2C): o VBHCD está alinhado à KBV, ou seja, enfatiza, na concepção da estratégia empresarial, que a fonte e a sustentabilidade de desempenho superior advêm de competências baseadas em conhecimento desenvolvidas por meio de um processo de contínuo aprendizado organizacional.

Destas proposições, deriva-se que as dimensões analíticas de interesse para este estudo são: Estratégia e Medidas de Desempenho (a partir de P1), e Fonte de Vantagem Competitiva e Fonte de Sustentabilidade de Vantagem Competitiva (para as três proposições alternativas P2a-P2c). As dimensões Mercado (que têm características que são diferentemente pressupostas de acordo com cada teoria de estratégia) e Unidade de Análise (e eventualmente outros subelementos conceituais de cada modelo) também foram incluídas por serem úteis para corroborar uma das proposições alternativas P2a-P2c.

Os textos analisados foram os trabalhos seminais do VBHCD (PORTER; TEISBERG, 2004, 2006) ou de seus artigos de maior divulgação (PORTER, 2008a, 2009). O objetivo foi mapear os fundamentos do modelo VBHCD e compará-los aos fundamentos das correntes teóricas de estratégia empresarial revisadas. As evidências são os constructos utilizados, os argumentos e as relações entabuladas por Porter e Teisberg $(2004,2006)$ e Porter $(2008 a, 2009)$ para explicar a base da melhoria do desempenho das organizações de saúde no seu modelo VBHCD. As dimensões de análise e suas respectivas caracterizações são apresentadas no Quadro 2.

Quadro 2. Dimensões utilizadas na análise do Modelo Value-Based Health Care Delivery

\begin{tabular}{|l|l|}
\hline Dimensão de Analise & Caracterização \\
\hline Estratégia & $\begin{array}{l}\text { Qual a importância da estratégia? Como é definida a estratégia no } \\
\text { modelo? }\end{array}$ \\
\hline
\end{tabular}




\begin{tabular}{|l|l|}
\hline Medidas de desempenho & $\begin{array}{l}\text { Qual(is) a(s) medida(s) de desempenho das organizações de saúde no } \\
\text { modelo? Incorpora outras medidas além do desempenho econômico- } \\
\text { financeiro? }\end{array}$ \\
\hline $\begin{array}{l}\text { Fonte de vantagem } \\
\text { competitiva }\end{array}$ & $\begin{array}{l}\text { Qual a fonte de vantagem competitiva? É baseada em posicionamento } \\
\text { ou em recursos e capacidades? Quais os seus mecanismos da fonte } \\
\text { da vantagem competitiva? Quais as opções de posicionamento (se } \\
\text { aplicável)? }\end{array}$ \\
\hline $\begin{array}{l}\text { Sustentação de vantagem } \\
\text { competitiva }\end{array}$ & $\begin{array}{l}\text { Qual a fonte de sustentabilidade da vantagem competitiva? Quais os } \\
\text { seus mecanismos? }\end{array}$ \\
\hline Mercado & $\begin{array}{l}\text { O mercado é definido como estático ou dinâmico? O papel da } \\
\text { competição? }\end{array}$ \\
\hline Unidade de análises & $\begin{array}{l}\text { Qual a principal unidade de análise do modelo? A indústria, a firma ou } \\
\text { ambas? }\end{array}$ \\
\hline
\end{tabular}

\section{O MODELO VALUE-BASED HEALTH CARE DELIVERY}

No modelo VBHCD, para Porter e Teisberg $(2004,2006)$, a fim de melhorar o resultado do setor de saúde, todos os participantes dos sistemas deveriam estar centrados em aumentar o valor para o cliente. Valor, neste contexto, diz respeito a resultados médicos ou de saúde, e eles devem sempre ser acompanhados de uma avaliação do gasto incorrido para alcançá-los. Ou seja, os resultados devem ser medidos com base em resultados clínicos (ou na saúde) alcançados por gasto financeiro.

Os autores defendem que bases erradas na forma de competir têm levado ao mau desempenho das empresas do setor. As perdas em termos de eficiência têm ocorrido em função de uma concentração excessiva, e de forma errada, em custos. Para os autores, minimizar custos é uma meta equivocada, que leva a resultados contraproducentes. Eliminar desperdícios e serviços desnecessários é benéfico, mas a verdadeira economia de custos deve decorrer de eficiências verdadeiras, não de transferência de custos, restrições nos cuidados (racionamento) ou redução da qualidade, segundo eles.

No modelo VBHCD, destacam-se alguns elementos relevantes para esta análise: (1) o valor da competição: Porter e Teisberg defendem a competição como forma de melhorar o setor. Eles não advogam qualquer forma de controle governamental da oferta de serviços. Apontam que o conteúdo atual da competição está errado: deveria ser direcionado para a competição por valor para o paciente, em vez da competição por custo. A competição em custos ou commoditização na saúde levou paradoxalmente à perda de eficiência, segundo eles, e o foco no valor para o paciente traria a tão desejada eficiência; (2) a qualidade defendida por Porter e Teisberg deverá ser medida e disseminada em termos de seus resultados (de saúde). Eles consideram que, embora desejável, a qualidade como conformidade dos processos não é o atributo que trará os benefícios almejados. A qualidade deveria ser medida em função de seus resultados de saúde, os quais deveriam ser amplamente divulgados. Os resultados de saúde em determinadas linhas de cuidados deveria ser à base da competição; (3) o critério de avaliação para o setor deveria ser o valor para o paciente medido em resultado por dólar gasto (gasto financeiro); (4) a liderança dos médicos nos processos: os autores colocam claramente que a mudança no setor deve ser liderada pelos médicos (em oposição a uma tendência atual de microgerenciar a atividade médica).

O modelo estimula o desenvolvimento de uma cultura organizacional que preconiza agregar valor para o paciente e este deve sempre ser medido em termos de resultados em termos de saúde. A estratégia das organizações se basearia na especialização em determinadas condições de saúde, em cujo nível deve ocorrer a competição entre organizações de saúde, com amplitude regional, nacional ou mesmo internacional. Como consequência desta especialização, a estruturação das empresas de saúde deve se dar em Linhas de Serviço ou Unidades de Práticas Integradas (UPI) para as respectivas condições de saúde. A coordenação dos processos deve se dar pelo ciclo completo de atendimento (o que incorpora uma visão de mais longo prazo para o resultado do tratamento das 
condições de saúde); a cobrança e o pagamento também devem ser por ciclo de atendimento; a avaliação deve mensurar os resultados clínicos ajustados pelo risco e pelos valores gastos (ambos medidos em todo o ciclo de atendimento e por condição de saúde).

\section{ANÁLISE DOS FUNDAMENTOS DO VBHCD}

Iniciando a análise a partir da dimensão Estratégia, percebe-se que o modelo VBHCD é fundamentalmente voltado para a estratégia das organizações de saúde e para os resultados do desempenho das organizações como um todo. É explicitado como um modelo que pretende trazer inovação nas estratégias e na organização geral das empresas atuantes neste setor (PORTER; GUTH, 2012; PORTER; PABO; LEE, 2013). É uma abordagem ampla que pretende modificar, o que no entender deles, estar organizado em torno de tradições e preferências dos médicos, em vez de em função do valor para o paciente (PORTER; TEISBERG, 2006). Esta abordagem ampla é o passo mais importante para a estruturação das organizações de saúde, dando à estratégia a mesma importância e definição que se tem nos estudos de estratégia empresarial. Uma passagem que ilustra esta visão no modelo descreve que...

Não haverá necessidade de predeterminar a melhor forma de estruturar prestadores e planos de saúde, de especificar os processos de tratamento a serem usados, de ditar como os sistemas de TI devem ser projetados ou que novas tecnologias devem ser adotadas. Se todos os participantes do sistema tiverem que mensurar e relatar resultados e competir por cliente ou paciente, o valor eleverá e as inovações irão proliferar. (PORTER; TEISBERG, 2007, p. 276).

Analisando-se a partir da dimensão Medidas de Desempenho, observa-se que o modelo VBHCD tem uma preocupação em torno de resultado econômico, mas também com o valor para o paciente. Porter e Teisberg, de forma central em seu modelo, lançam seu critério de avaliação do sistema que incorpora valor para paciente juntamente com gasto (valor medido em resultado por unidade monetária despendida), derivando, portanto, para valor/gastos como indicador de desempenho. Desta forma, o modelo não tem somente uma avaliação unidimensional do resultado das empresas, algo certamente diferente do mainstream da Estratégia, que preconiza a maximização de uma funçãoutilidade isolada (VASCONCELOS; CYRINO, 2000), tais como o valor de mercado total de longo prazo da firma (JENSEN, 2002) ou o valor presente líquido dos lucros da empresa (GRANT, 2002). Por outro lado, o VBHCD, neste aspecto, encontra consonância com outras evidências que demonstram que a gestão dos demais stakeholders primários (como os clientes, ou no caso, pacientes) está associada a aumento de valor da empresa (HILLMAN; KEIM, 2001) e com as proposições ligadas aos aspectos institucionais citados no referencial teórico de estratégia em saúde.

A própria concepção de valor para os pacientes (ou resultados de saúde) também é multidimensional e inclui a autonomia do paciente, tempo de vida, qualidade de vida e tempo de recuperação, dores, complicações, erros médicos, entre outros. Igualmente, aspectos ligados a valores pessoais dos pacientes podem ser importantes em áreas como efeitos colaterais, agressividade do tratamento e necessidade de internação (PORTER; TEISBERG, 2004, 2006). O modelo insiste que o valor deve ser medido, avaliado e disseminado pelos seus resultados para a saúde, além de outros indicadores. A mensuração da melhora efetiva da condição do paciente é a informação relevante e não somente se uma organização sob análise se ateve aos processos corretos ou recomendados. Sobre a relação entre essas dimensões, advogam que a melhoria em termos de valor levará a ganhos em termos de eficiência.

Com relação à importância do papel da Estratégia no VBHCD, o que se extrai dos textos é que ele está em linha com os conceitos originais (em Estratégia Empresarial) de Porter, que advoga que a efetividade operacional (que inclui, mas não se limita à eficiência) não pode ser considerada a estratégia da organização (PORTER, 1996). É necessária uma estratégia que diferencie a empresa das demais. Nos textos do modelo VBHCD, observa-se:

Dedicar-se a melhorar a eficácia operacional é importante em qualquer organização, mas não basta. Toda empresa precisa de uma estratégia norteadora, que defina objetivos e propósitos, o negócio ou negócios em que vai operar, os serviços que irá oferecer e de que maneira tentará se distinguir de seus pares. Sem uma estratégia, uma organização carece de clareza para atingir a verdadeira excelência. (PORTER; TEISBERG, 2007, p. 140). 
Em relação à estratégia em si, os autores defendem que resultados excelentes levariam a mais pacientes, maior eficiência e margens mais altas (PORTER; TEISBERG, 2006). Os mecanismos pelos quais isso ocorre se assemelham ao que Porter descreve como posicionamento: "médicos deveriam competir para serem os melhores na abordagem de um determinado conjunto de condições de saúde ou num determinado segmento de pacientes, ou ambos" (PORTER; TEISBERG, 2007, p. 55). E, ainda, "... [à] medida que os hospitais e os grupos de médicos se tornam mais parecidos, eles perdem o foco estratégico necessário para alcançar a verdadeira excelência" (PORTER; TEISBERG, 2007 , p. 58). O entendimento dos autores é que a especialização no atendimento a determinadas condições de saúde, os investimentos necessários para isso, a experiência a ser adquirida, os ciclos de aprendizado e todo os demais aspectos carregam em si uma escolha estratégica com grandes trade-offs, e que somente podem ser imitados com grandes custos por parte dos concorrentes.

Nos textos originais de Porter (1986), o posicionamento é entendido como a escolha de um grupo em que competir, e grupo estratégico é entendido como um grupo de empresas, em uma indústria, que estão seguindo uma estratégia idêntica ou semelhante ao longo de dimensões estratégicas. Nos textos do VBHCD, Porter e Teisberg descrevem algumas escolhas que podem ser compreendidas como decisões de posicionamento:

Existem inúmeras maneiras pelas quais um prestador pode se distinguir numa unidade de prática: concentrando-se em diagnósticos complexos; servindo a grupos específicos de pacientes, como mulheres ou idosos, com condições co-ocorrentes; oferecendo extraordinária tempestividade ou eficiência; demonstrando excelência no gerenciamento de doenças no longo prazo; e outras. (PORTER; TEISBERG, 2007, p. 160).

Para os autores, os participantes do setor da saúde definiram equivocadamente o seu negócio. A competição atualmente ocorre em linhas amplas de serviços, e não nos serviços individuais (em que, segundo os autores, deveria ocorrer). Os prestadores oferecem todos os serviços possíveis e se dispõem a tratar de qualquer paciente que os procure. Os planos de saúde contratam prestadores de todo tipo. No entanto, segundo Porter e Teisberg, a amplitude dos serviços oferecidos tem pouco impacto no valor para o paciente. O que importa é a capacidade do prestador de entregar valor em cada condição de saúde específica: "o sistema está estruturado como se a assistência à saúde fosse um único negócio (linha de serviço), em vez de muitos negócios diferentes; como se os prestadores fossem todos equivalentes, os resultados fossem sempre os mesmos e todos os pacientes tivessem as mesmas preferências" (PORTER; TEISBERG, 2007, p. 55).

Segundo eles, portanto, os participantes deveriam estruturar a prestação de serviços a fim de fornecer um atendimento verdadeiramente integrado durante todo o ciclo de atendimento em uma condição específica (PORTER; TEISBERG, 2006). O mecanismo de expansão dos negócios ocorreria quando prestadores com excelência numa condição de saúde crescem geograficamente, gerenciando serviços em vários locais e, assim, alavancando escala, especialização na condição, métodos de prestação dos serviços de saúde, treinamento de equipes, sistemas de mensuração e reputação (PORTER; TEISBERG, 2004, 2006).

Outro conceito, o da fronteira de produtividade, utilizado nos textos iniciais de Porter (1996), também é identificável como explicação para a existência de uma grande margem para melhorias em qualidade e custo, simultaneamente e em curto prazo, no Setor de Saúde. Para Porter e Teisberg $(2004,2006)$, só implementando as melhores práticas já conhecidas, virtualmente todos os prestadores podem melhorar tanto a qualidade quanto as margens de lucro, sem elevar os preços. Para Porter (1996), em seus trabalhos sobre Estratégia Empresarial, o conceito é utilizado para demonstrar que existem falsos trade-offs e para fomentar aquele que seria o verdadeiro trade-off relevante para as empresas: a adaptação entre as atividades a fim de implementar um determinado posicionamento.

Como se percebe, os fundamentos para as explicações acima se alinham à teoria porteriana para estratégia desenvolvida por volta dos anos oitenta. Porém, por outro lado, explicações típicas da VBR para fonte de vantagem competitiva também são utilizadas como argumentos por Porter e Teisberg. Ou seja, eles usam como fundamentos para o seu modelo VBHCD argumentos baseados não somente nas ideias originais de Porter sobre estratégia empresarial, mas também em ideias de outra linha concorrente de explicação da estratégia e do desempenho das organizações, a VBR. Isto é observável no trecho a seguir, em que se faz uma clara alusão ao argumento que recursos e capacidades devem dirigir as escolhas em estratégia: 
Parte da escolha estratégica das linhas de serviço advém da análise de equiparação entre a complexidade e acuidade das condições diagnosticadas e tratadas com as habilidades, os recursos tecnológicos, as instalações e a base de custos da instituição. Serviços simples ou de rotina não deveriam ser oferecidos por instituições que não puderem realizá-los a um custo competitivo. Em oposição, serviços complexos e fora do comum não deveriam ser oferecidos por instituições que carecem de experiência, escala e capacidades para alcançar resultados excelentes. (PORTER; TEISBERG, 2007, p. 147).

Os autores engendram, portanto, argumentos de que o desempenho é consequência de uma combinação de recursos e capacidades: "prestadores têm que escolher o conjunto de condições de saúde nos quais eles são capazes de alcançar excelência em termos de valor ao paciente, dada a sua combinação particular de pacientes, habilidades e outras circunstâncias" (PORTER; TEISBERG, 2007 , p. 147). Essas últimas descrições mostram, de forma clara, uma preocupação em analisar não somente o ambiente, mas também as firmas internamente.

Há também descrições sobre culturas organizacionais difíceis de imitar, conceito presente na VBR como mecanismo de sustentação da vantagem competitiva: "os melhores prestadores continuaram a apresentar desempenho acima da média. Suas atividades na prestação de serviços de saúde e suas culturas organizacionais são difíceis de replicar" (PORTER; TEISBERG, 2007, p. 103).

Além dessas citações, os textos de Porter e Teisberg são repletos de argumentos para demonstrar que não será necessário pagamento mais alto para recompensar melhores resultados. Prestadores excelentes com frequência alcançariam melhores margens, valendo-se de maior eficiência, um mecanismo explicativo da VBR para desempenho acima da média. Isso ocorreria "porque os prestadores excelentes podem expandir as vantagens de que desfrutam valendo-se de aprendizagem maior e eficiência melhor" (PORTER; TEISBERG, 2007, p. 90).

O conhecimento e o aprendizado, temas virtualmente ausentes no paradigma porteriano e mais ligados a RBV/KBV, são centrais para o modelo VBHCD: "o desenvolvimento de conhecimento é um elemento habilitador fundamental na competição baseada em valor" (PORTER; TEISBERG, 2007, p. 182).

Porter e Teisberg descrevem claramente o que se pode considerar como rotinas (organizacionais) para aprender, um dos principais componentes das capacidades organizacionais (DOSI, NELSON, WINTER, 2000):

O desenvolvimento sistemático do conhecimento consiste em, pelo menos, três componentes: medir a analisar resultados, identificar as melhorias de processos e treinar o quadro de pessoal. O esforço precisa de um gerenciamento contínuo por parte dos médicos e auxiliares competentes trabalhando juntos como uma equipe sob uma liderança envolvida. Requer uma abordagem estruturada, orientada para dados. Medir e sistematizar todos os quatro níveis de dados na hierarquia $[. .$.$] . As unidades de prática integradas precisam destinar tempo a reuniões regulares$ para proceder a análise crítica de resultados, examinar as causas de questões problemáticas, explorar soluções possíveis, e aprender [...]. (PORTER; TEISBERG, 2007, p. 191).

O que os autores descrevem como os elementos do círculo virtuoso que leva a melhoria de resultados no VBHCD nada mais são do que o ganho de experiência, a escala e o aprendizado, pois a experiência dos prestadores (só perdendo para os resultados propriamente ditos) é uma das informações mais relevantes para apoiar a competição baseadas em valor, segundo os textos (PORTER, 2009; PORTER; TEISBERG, 2006). Métodos (processos) e atributos dos pacientes são informações hierarquicamente inferiores neste sentido (PORTER; TEISBERG, 2006). E a experiência, como se sabe, um elemento equivalente ao que se define como conhecimento tácito ou conhecimento procedural (KOGUT; ZANDER, 1992), tem sido descrita como fonte de evolução das rotinas organizacionais e desempenho superior, por autores ligados ao MCD (ZOLLO; WINTER, 2002). Além disso, o resultado é fruto de um trabalho coletivo: "isso ocorre porque valor não é criado por cada especialidade individual, mas pela atividade coletiva de toda uma equipe" (PORTER, 2008a, p. 505). Neste trabalho, a coordenação à comunicação da equipe do prestador assume papel fundamental (PORTER; TEISBERG, 2006). Ou, ainda, "... valor é maximizado pelas equipes dedicadas em que pessoas trabalham diariamente em conjunto para melhorar suas habilidades para responder a um conjunto particular de problemas, reconhecendo que é o seus esforços coletivos que determinam o resultado global" (PORTER, 2008a, p. 507).

A inovação, outro elemento ligado à RBV/KBV, também virtualmente ausente no paradigma porteriano, é um dos oito princípios fundamentais no VBHCD. A inovação deve ser fomentada: "a 
trajetória de inovação tem sido desviada, pelos incentivos do atual sistema, para medicamentos e dispositivos. Imensas oportunidades para melhorar a organização, os métodos, as instalações e a coordenação na prestação dos serviços de saúde apenas começaram" (PORTER; TEISBERG, 2007, p. 131).

Ainda sobre inovação, ressalta-se o fato que ela deve ocorrer, segundo os autores, em novos métodos, novas instalações, novas estruturas organizacionais, novos processos ou novas formas de colaboração entre prestadores (PORTER; TEISBERG, 2006), ou seja, claramente em elementos intra-organizacionais. Além disso, a ênfase em inovações constantes demonstra uma visão dinâmica de mercado no VBHCD.

Concentrando-se na dimensão analítica de Mercado, observa-se que, já nas partes iniciais dos textos, os autores reiteram o papel da competição como mecanismo para a melhoria de qualquer setor e, consequentemente, também da área da saúde. Eles descrevem que a competição entre rivais do setor privado, ao longo da história, sempre foi uma grande impulsionadora de melhorias na qualidade e dos custos de produtos e serviços (PORTER; TEISBERG, 2004, 2006).

Há ainda nos textos várias descrições de como ocorrem os mecanismos econômicos subjacentes, principalmente aqueles relacionados a ganhos de escala. Para os autores, muitos prestadores com escala insuficiente carecem das capacidades necessárias para alcançar a excelência (PORTER; TEISBERG, 2004, 2006). Para eles, poucos prestadores percebem que a busca de amplitude em serviços não relacionados ou frouxamente relacionados acarreta um grande custo de oportunidade, pois dispersa o foco e os investimentos que poderiam ser usados para eles se tornarem verdadeiramente excelentes e crescerem em condições de saúde específicas (PORTER; TEISBERG, 2006). Segue uma destas descrições:

\begin{abstract}
Assistência a saúde não se torna mais eficiente só porque um hospital trata o dobro de pacientes numa ampla faixa de doenças; os pacientes continuam sendo tratados um de cada vez e de acordo com suas circunstâncias particulares. Qualquer economia de escala significativa na prestação de um serviço de assistência à saúde depende das peculiaridades das condições de saúde e respectivos tratamentos [...]. Portanto, descontos indiscriminados com o intuito de atrair um grande grupo não aumentam o valor e sim diminuem a renda dos hospitais e médicos. Além do mais, isso gera uma grande pressão nos profissionais de saúde para que atendam a mais clientes por dia e apressem o tempo de atendimento para recuperar os lucros. (PORTER; TEISBERG, 2007, p. 49).
\end{abstract}

Portanto as principais economias de escala na prestação de serviços de saúde se dão em linhas de serviço, e não para o hospital como um todo. Ainda nesta linha de raciocínio, eles consideram que, com raras exceções, as fusões de hospitais resultaram em pouco ou nenhuma consolidação e integração de fato no nível de linha de serviço (PORTER; TEISBERG, 2006).

Em relação à gestão dos processos, os autores acreditam que, em vista das muitas variáveis e julgamentos envolvidos nos tratamentos médicos, é possível que os prestadores variem bastante em seus resultados, mesmo que sejam uniformes quanto ao cumprimento dos processos (PORTER; TEISBERG, 2004, 2006). Por isso que eles defendem que, em vez de medir os resultados e recompensar os prestadores excelentes com mais pacientes, a abordagem atual tem sido indiscriminada, tentando elevar todos os prestadores de um serviço a um nível aceitável. As principais ferramentas têm sido diretrizes para a prática e normas de atendimento, que todos os prestadores têm que atender, e iniciativas quanto à qualidade, na sua maioria, não são de fato sobre qualidade (resultados), mas sobre processos. A maioria dos programas de "pagamento por desempenho" consiste, na verdade (segundo os autores), em pagamento por cumprimento de normas (PORTER; TEISBERG, 2006).

Segue, no Quadro 3, uma síntese dos achados e o seu cotejamento com as proposições elaboradas.

Quadro 3. Síntese dos achados e interpretação da análise do Modelo Value-Based Health Care Delivery

\begin{tabular}{|l|l|l|}
\hline $\begin{array}{l}\text { Dimensão de } \\
\text { Analise }\end{array}$ & Achados & Interpretação \\
\hline Estratégia & $\begin{array}{l}\text { Definição do negócio ("nível de } \\
\text { competição") e da estratégia como } \\
\text { etapas iniciais mais importantes. }\end{array}$ & Suporta H1 \\
\hline
\end{tabular}




\begin{tabular}{|l|l|l|}
\hline $\begin{array}{l}\text { Medidas de } \\
\text { desempenho }\end{array}$ & $\begin{array}{l}\text { Valor para paciente (medida } \\
\text { multidimensional) e custos/gastos. }\end{array}$ & Suporta H1 \\
\hline $\begin{array}{l}\text { Fonte de vantagem } \\
\text { competitiva }\end{array}$ & $\begin{array}{l}\text { Posicionamento e uso de recursos } \\
\text { e capacidades valiosos e raros, } \\
\text { principalmente recursos baseados no } \\
\text { conhecimento. }\end{array}$ & $\begin{array}{l}\text { Usa elementos de duas teorias } \\
\text { concorrentes de estratégica. Não } \\
\text { explicitação de contradições }\end{array}$ \\
\hline $\begin{array}{l}\text { Sustentação de } \\
\text { vantagem competitiva }\end{array}$ & $\begin{array}{l}\text { Trade-offs importantes e uso de } \\
\text { recursos e capacidades de difícil } \\
\text { imitação. }\end{array}$ & $\begin{array}{l}\text { Usa elementos de duas teorias } \\
\text { concorrentes de estratégica. Não } \\
\text { explicitação de contradições }\end{array}$ \\
\hline Mercado & $\begin{array}{l}\text { Competição e inovação com papel } \\
\text { importante }\end{array}$ & $\begin{array}{l}\text { Usa elementos de duas teorias } \\
\text { concorrentes de estratégica. Não } \\
\text { explicitação de contradições }\end{array}$ \\
\hline Unidade de análises & A indústria e a firma & $\begin{array}{l}\text { Usa elementos de duas teorias } \\
\text { concorrentes de estratégica. Não } \\
\text { explicitação de contradições }\end{array}$ \\
\hline
\end{tabular}

\section{DISCUSSÃO}

Como se pode perceber, a partir dos textos do VBHCD analisados, o modelo se utiliza de mecanismos causais baseados em fundamentos econômicos de mais de uma corrente estratégica. Diferente do que se poderia esperar, o modelo VBHCD não se baseia exclusivamente no paradigma porteriano em Estratégia Empresarial. Esse é o fato mais marcante desta análise, dado que Michael Porter, seguidas vezes, critica a VBR, seja de forma explícita (STONEHOUSE; SNOWDON, 2007) ou implícita (PORTER, 1996). O fato de importar mecanismos causais de diferentes correntes teóricas aumenta a complexidade e a responsabilidade de sua utilização por parte dos pesquisadores, além de expor possíveis contradições.

No modelo VBHCD, há mecanismos causais explicando o desempenho das empresas de saúde que podem ser associados tanto a uma posição de mercado específica, quanto à utilização de recursos e capacidades das organizações. Encontram-se também argumentos ligados ao aprendizado, ao conhecimento e à inovação, temas praticamente ausentes nos trabalhos de Porter dos anos 70 e 80, quando ele começou a publicar sobre o modelo de posicionamento. Esses últimos elementos, bem como a aplicação de melhores práticas, são todos ligados à RBV/KBV.

Diversos autores acreditam que há uma tendência de maior integração entre as principais teorias concorrentes no campo de estratégia (HOSKINSSON et al., 1999; RAY; BARNEY; MUHANNA, 2004; SPANOS; LIOUKAS, 2001). Por outro lado, essa integração não pode ser feita superficialmente e sua complexidade aparece quando se aprofunda a discussão acerca de conceitos relevantes e do lócus da vantagem competitiva.

A pesquisa em estratégia parece não ter sido capaz ainda de propor tal estrutura que possa integrar ambas as teorias, em função da complexidade das duas abordagens, que exige a consideração de inúmeros aspectos, que algumas vezes se apresentam concorrentes entre as duas visões (WALTER; BANDEIRA-DE-MELLO; GÖTZE, 2005).

Existem marcantes e concorrentes diferenças entre as duas teorias, como exposto anteriormente e sintetizado na Tabela 1. Enquanto a teoria porteriana sobre Estratégia se concentra na análise da indústria, a VBR se concentra na análise interna das firmas. Na teoria porteriana, as necessidades internas das firmas são uma consequência da análise externa, isto é, da observação do mercado; enquanto que na VBR é a partir de uma dada base de recursos da firma que são definidos em quais mercados será interessante atuar. Nesse sentido, pesquisadores da VBR fazem da análise interna de forças e fraquezas o ponto de partida para desenvolvimento da estratégia; enquanto modelos de análise ligados à teoria de Porter são mais úteis para avaliar oportunidades e ameaças. Nesta última também, as necessidades de recursos e competências são consequência dos fatos do mercado; enquanto que na VBR eles são a base para definição do posicionamento (HOSKINSSON et al., 1999; SPANOS; LIOUKAS, 2001). 
Os pressupostos das duas principais correntes em Estratégia são fundamentalmente distintos: homogeneidade e mobilidade dos recursos - no caso do paradigma porteriano -, heterogeneidade e imobilidade dos recursos - para a RBV/KBV. As diferentes teorias são alicerçadas sobre seus respectivos pressupostos, com os quais elas têm consistência, e a partir dos quais são desenvolvidas todas as suas implicações. Em função disso, cada uma dessas teorias é aplicável - ou seja, tem melhor aderência - a distintos contextos.

Porter e Teisberg $(2004,2006)$ não explicitam esses eventuais conflitos entre os pressupostos, nem o fato de, no seu modelo, ocorrer uma tentativa de integração entre perspectivas teóricas com distintos fundamentos. A não explicitação destes aspectos pode levar ao que se chama de pragmatismo (POWELL, 2001), de apenas usar as teorias como guias úteis para a prática, independente de serem formalmente válidas. Para Durand (2002), o pragmatismo pode levar ao risco do relativismo, que, por sua vez, pode desconectar as teorias da realidade gerencial. $\mathrm{O}$ relativismo pode obstruir o desenvolvimento das teorias e, segundo ele, tornar a busca pela verdade um mero jogo de palavras.

O VBHCD parece adotar esta visão integradora das teorias de estratégia. Os autores assumem que existe uma grande necessidade de transformação da prática e o que traria retorno de fato teria caráter mais normativo, que é encontrar maneiras de melhorar o atendimento (PORTER; TEISBERG, 2006). Porém, a discussão e o conhecimento dos mecanismos subjacentes do modelo são úteis à medida que permitem maior capacidade de adaptação a diferentes contextos para sua aplicação.

Nada impede que planejamentos orientados pelo mercado e pelos processos possam ser paralelamente aplicados. A avaliação de oportunidades e ameaças ambientais pode ser realizada ao se aplicar técnicas do paradigma porteriano, como os modelos das cinco forças de Porter e as estratégias genéricas; e a VBR pode contribuir para a análise de forças e fraquezas, na medida em que avalia os recursos e as competências estrategicamente relevantes para uma firma. Contudo, quando se apresenta um modelo prescritivo, baseado em estudos científicos, como é o caso do modelo VBHCD, sua coerência interna deve ser analisada e formalmente validada.

\section{CONCLUSÃO}

O VBHCD é um modelo formulado especificamente para a área da saúde, que incorpora aspectos peculiares a ela, principalmente no que diz respeito à forma como é realizada a avaliação de seu desempenho. Por outro lado, o objetivo do trabalho não foi dar suporte, ou mesmo justificar, a sua aplicação no setor - um esforço que usualmente cabe aos seus autores ou seguidores. $O$ artigo tratase, na verdade, de uma análise das vertentes teóricas - não explicitadas - dos seus pressupostos. Esta análise, à luz da literatura sobre os fundamentos econômicos da vantagem competitiva, revela que o VBHCD não explicita a utilização de diferentes mecanismos causais oriundos de diferentes e concorrentes teorias aparentemente contraditórias. Desta forma, ele acaba por inserir-se no debate atual da pesquisa em estratégia empresarial, pois pode ser visto como uma forma de integrar essas correntes, ainda que inconscientemente pelos seus autores. Essa integração, porém, também não está explícita nos textos de Porter e Teisberg (2004, 2006).

Para concluir, e retomando uma visão mais ampla, é importante sempre manter em mente que as organizações da área de saúde, principalmente os hospitais, não têm seu desempenho medido somente por seus aspectos econômicos e financeiros, pois como lembram os autores, "resultados financeiros são uma consequência, não uma meta em si e por si mesma. Uma boa margem de superávit operacional não pode compensar a mediocridade no atendimento [aos pacientes]" (PORTER; TEISBERG, 2007, p. 144).

\section{REFERÊNCIAS}

ACEDO, F. J.; BARROSO, C.; GALAN, J. L. The resource-based theory: dissemination and main trends. Strategic Management Journal, v. 27, n. 7, p. 621-636, 2006.

ALEXANDER, J. A.; D'AUNNO, T. A. Alternatives Perspectives on Institutional and Market Relationships in the U.S. Health Care Sector. In: MICK, S. S.; WYTTENBACH M. E. Advances in Health Care Organization Theory. San Francisco: Jossey-Bass, 2003. 
ALMEIDA, C. Debate sobre o artigo de Hillegonda Maria Dutilh Novaes. Cad. Saúde Pública, Rio de Janeiro, v. 20, Sup. 2, p. 158-159, 2004.

ALMeIDA, M. F. Cad. Saúde Pública, Rio de Janeiro, 20 Sup 2:S147-S173, 2004.

BANDEIRA-DE-MELLO, R.; MARCON, R. Avaliação da eficácia das estratégias de posicionamento e da atratividade setorial, do ponto de vista do acionista. Revista de Administração Contemporânea, v. 8, n. 2, p. 27-50, 2004.

BANDEIRA-DE-MELLO, R.; MARCON, R. Unpacking firm effects: modeling political alliances in variance decomposition of firm performance in turbulent environments. Brazilian Administration Review, v. 2, n. 1, p. 21-37, 2005.

BARNEY, J. Firm resources and sustained competitive advantage. Journal of Management, v. 7, n. 1, p. 99-120, 1991.

BARNEY, J. How a firm's capabilities affect boundary decisions. Sloan Management Review, Spring 1999.

BARNEY, J. B. Is the Resource-Based Theory a Useful Perspective for Strategic Management Research? Yes. Academy of Management Review, v. 26, n.1, p. 41-56, 2001.

BRITO, L. A. L.; VASCONCELOS, F. C. The variance composition of firm growth rates and competitive advantage. Brazilian Administration Review, v. 6, n. 2, p. 118-136, April/June 2009.

CAVES, R.; PORTER, M. From entry barriers to mobility barriers: conjectural decisions and contrived deterrence to new competition. Quarterly Journal of Economics, v. 91, p. 241-261, 1977.

CHRISTENSEN, C. M.; GROSSMAN, J. H.; HWANG, J. The Innovator's Prescription: A Disruptive Solution for Health Care. New York: McGraw-Hill, 2009.

DIERICKX, I.; COOL, K. Asset stock accumulation and sustainability of competitive advantage. Management Science, v. 35, n.12, December 1989.

DOSI, G.; NELSON, R.; WINTER, S. The nature and dynamics of organizational capabilities. New York: Oxford University Press, 2000.

DUBIN, R. Theory Development. New York: Free Press, 1978.

DURAND, R. Competitive advantages exist: A critique of Powell. Strategic Management Journal, v. 23, n. 9, September 2002.

GRANT, R. Contemporary strategy analysis: concepts, techniques, applications. Oxford: Blackwell Publishing, 2002.

HAMEL, G.; PRAHALAD, C. K. Competing for the Future. Boston: HBRP, 1994.

HAWAWINI, G. ; SUBRAMANIAN, V.; VERDIN, P. Is performance driven by industry -or firm-specific factors? A newlook at the evidence. Strategic Management Journal, v. 24, n. 1, p. 1-16, 2003.

HILLMAN, A.; KEIM, G. Shareholder value, stakeholder management, and social issues: What's the bottom line? Strategic Management Journal, v. 22, n. 2, p. 125-139, February 2001.

HOSKISSON et al. Theory and research in strategic management: swings of a pendulum. Journal of Management, v. 25, p. 417-456, 1999.

IBGE. Disponível em: http://www.ibge.gov.br/home/default.php. Acesso em: 8 jan. 2012.

JENSEN, M. Value maximization, stakeholder theory, and the corporate objective function. Business Ethics Quarterly, v. 12, n.1, 2002.

KOGUT, B. ; ZANDER, U. Knowledge of the firm, combinative capabilities, and the replication of technology. Organization Science, v. 3, n. 3, 1992.

KOTLER, P.; SHALOWITZ, J.; STEVENS, R. J. Strategic marketing for health care organizations: building a customer-driven health system. Hoboken, NJ: Jossey-Bass, 2008. 
LUKE, R. D; BEGUN, J. W. ; WALSTON, S. Strategy Making in Health Care Organizations. In: SHORTELL, S. M.; KALUZNY, A. D. Health Care Management: Organization, Design and Behavior. Albany, NY: Delmar Thomson Learning, 2000.

LUKE, R. D; WALSTON, S. Strategy in an Institutional Environment: Lessons Learned from the 1990s "Revolution" in Health Care. In: MICK, S. S.; WYTTENBACH, M. E. Advances in Health Care Organization Theory. San Francisco: Jossey-Bass, 2003.

MCGAHAN, A. M; PORTER, M. E. How much does industry matter, really? Strategic Management Journal, v. 18, n. 6, 1997.

MINISTÉRIO DA SAÚDE. Cadastro Nacional de Estabelecimentos de Saúde. Disponível em: http://cnes. datasus.gov.br/Index.asp. Acesso em: 8 jan. 2012.

MINTZBERG, H. Managing the miths of health care. World Hospitals and Health Services, v. 48, n.3, p. 4-7, 2012.

MISANGYI et al. A new perspective on a fundamental debate: a multilevel approach to industry, corporate, and business unit effects, Strategic Management Journal, v. 27, n. 6, p. 571-590, 2006.

MISOCZKY et al. Fórum Administração e Saúde. Revista de Administração de Empresas, v. 49, n. 4, Out-Dez 2009.

NOVAES, H. M. D. Pesquisa em, sobre e para os serviços de saúde: panorama internacional e questões para a pesquisa em saúde no Brasil. Cadernos de Saúde Pública, Rio de Janeiro, 20 Sup 2, S147-S173, 2004.

PAIM, J. S.; TEIXEIRA, C. F. Política, planejamento e gestão em saúde: balanço do estado da arte. Rev. Saúde Pública, v. 40, N. Esp, p. 73-78, 2006.

PETERAF, M. The cornerstones of competitive advantage: a resource-based view. Strategic Management Journal, v. 14, p. 179-191, 1993.

PETERAF, M.; BERGEN, M. Scanning dynamic competitive landscapes: a market-based and resourse-based framework. Strategic Management Journal, v. 24, p. 1027-1041, 2003.

PINHO, A.; SILVA, J. Posicionamento estratégico e desempenho de mercado da indústria farmacêutica à luz da tipologia de Porter. Revista de Administração Contemporânea, v. 5, n. 3, p. 27-52, 2001.

POLANCZIK, C. A. Pesquisa em serviços de saúde: precisamos valorizar essa tendência! Rev. HCPA, v. 30, n. 1, p. 3-4, 2010.

PRIEM, R. L.; BUTLER, J. E. Is the Resource-Based Theory a Useful Perspective for Strategic Management Research? Academy of Management Review, v. 26, n. 1, p. 22-40, 2001a.

PRIEM, R. L.; BUTLER, J. E. Tautology in the Resource-Based View and Implications of Externally Determined Resource Value: Further Comments. Academy of Management Review, v. 26, n. 1, p. 57-66, 2001b.

PORTER, M. E. The structure within industries and companies' performance. The Review of Economics and Statistics, v. 61, n. 2, p. 214-227, May 1979.

PORTER, M. E. The contributions of industrial organization to strategic management. Academy Of Management Review, v. 6, n. 4, p. 609-620, 1981.

PORTER, M. E. Estratégia competitiva: técnicas para análise de indústrias e da concorrência. Rio de Janeiro: Campus, 1986.

PORTER, M. E. What is Strategy? Harvard Business Review, p. 61-78, November-December 1996.

PORTER, M. E. Value-based Health Care Delivery. Annals of Surgery, v. 248, n. 4, p. 503-509, 2008 a.

PORTER, M. E. The Five competitive forces that shape strategy. Harvard Business Review, p. 1-18, January, 2008b.

PORTER, M. E. Health Care 2009: a strategy for health care reform - toward a value-based system. New England Journal of Medicine, v. 361, n. 2, p. 10-112, 2009. 
PORTER; M. E.; PABO, E. A.; LEE, T. H. Redesigning primary care: a strategic vision to improve value by organizing around patient's needs. Health Affairs, v. 32, n. 3, March 2013.

PORTER, M. E.; GUTH, C. Redefining German health care: moving to a value-based system. New York: Springer, 2012.

PORTER, M. E.; Teisberg, E. O. Redefining Competition in Health Care. Harvard Business Review, June 2004.

PORTER, M. E.; TEISBERG, E. O. Redefining health care: creating value-based competition on results. Boston, MA: Harvard Business School Press, 2006.

PORTER, M. E.; TEISBERG, E. O. Repensando a saúde: estratégias para melhorar a qualidade e reduzir os custos. Porto Alegre: Bookman, 2007.

POWELL, T. Competitive advantage: Logical and philosophical considerations. Strategic Management Journal, v. 22, n. 9, p. 875-888, September 2001.

PRAHALAD, C.; HAMEL, G. The core competence of the corporation. Harvard Business Review, p. 7991, May/june 1990.

RAMOS-RODRIGUEZ, A. R.; RUIZ-NAVARRO, J. Changes in the intellectual structure of strategic management research: a bibliometric study of the Strategic Management Journal, 1980-2000, Strategic Management Journal, v. 25, n.10, p. 981-1004, 2004.

RAY, G.; BARNEY, J.; MUHANNA, W. Capabilities, business processes, and competitive advantage: choosing the dependent variable in empirical tests of the resource-based view. Strategic Management Journal, v. 25, p. 23-37, 2004.

RUMELT, R. P. How much does industry matter? Strategic Management Journal, v. 12, n. 3, p. 167$185,1991$.

SCHROEDER, R. G.; BATES, K. A.; JUNTTILA, M. A. A resource-based view of manufacturing strategy and the relationship to manufacturing performance. Strategic Management Journal, v. 23, n. 2, p. 105-117, 2002.

SCOTT, W. R. 2003. The Old Order Changeth: The Evolving World of Health Care Organizations. In: MICK, S. S; WYTTENBACH, M. E. Advances in Health Care Organization Theory. San Francisco: JosseyBass, 2003.

SCOTT, W. R. et al. Institutional Change and Healthcare Organizations: from Professional Dominance to Managed Care. Chicago: The University of Chicago Press, 2000.

SEHNEM, S.; LAZZAROTTI, F.; BANDEIRA-DE-MELLO, R. Uma análise longitudinal da utilização do paradigma porteriano no Brasil. Brazilian Business Review, v. 7, n. 2, p. 21-39, 2010.

SHORTELL, S. M.; ZAJAC, E. J. Health Care Organizations and the Development of Strategic-Management Perspective. In: MICK, S. S. et. al. Innovations in Health Care Delivery. San Francisco: Jossey-Bass, 1990.

SPANOS, Y.; LIOUKAS, S. An examination into the causal logic of rent generation: contrasting Porter's competitive strategy framework and the resource-based perspective. Strategic Management Journal, v. 22 , p. $907-934,2001$.

STONEHOUSE, G.; SNOWDON, B. Competitive Advantage Revisited: Michael Porter on Strategy and Competitiveness. Journal of Management Inquiry, v. 16, n. 3, p. 256-273, 2007.

TEECE, D; PISANO, G.; SCHUEN, A. Dynamic capabilities and strategic management. Strategic Management Journal, v. 18, n. 7, p. 509-533, 1997.

VASCONCELOS, F.; CYRINO, A. Vantagem competitiva: os modelos teóricos atuais e a convergência entre estratégia e teoria organizacional. Revista de Administração de Empresas, v. 40, n. 4, OutubroDezembro 2000.

VELOSO, G. G; MALIK, A. M. Análise do desempenho econômico-financeiro de empresas de saúde. RAEeletrônica, v. 9, n. 1, Art. 2, jan./jun 2010. 
WALTER, F.; BANDEIRA-DE-MELLO, R.; GÖTZE, U. A. Integração entre a Visão baseada em Recursos e a Visão baseada no Mercado da Vantagem Competitiva: dificuldades e perspectivas. IN: XXV ENCONTRO NACIONAL DE ENGENHARIA DE PRODUÇÃO, XXV, 2005, Anais... Porto Alegre, RS, Brasil. Outubro, 2005.

ZOLLO, M.; WINTER, S. Deliberate learning and the evolution of dynamic capabilities. Organization Science, v. 13, n. 3, p. 339-351, 2002. 